\title{
Multi-Stability and Super Cavity Solitons in Microresonator Frequency Combs
}

\author{
Tobias Hansson \\ Department of Applied Physics, Chalmers University of Technology, SE-41296 Göteborg, Sweden \\ Dipartimento di Ingegneria dell'Informazione, Università di Brescia, via Branze 38, 25123 Brescia, Italy \\ tobhan@chalmers.se \\ Stefan Wabnitz \\ Dipartimento di Ingegneria dell'Informazione, Università di Brescia and INO-CNR, via Branze 38, 25123 Brescia, \\ Italy \\ stefan.wabnitz@unibs.it
}

\begin{abstract}
Frequency comb generation in microresonators is considered beyond the meanfield approximation. We show the appearance of multi-valued stationary states and predict the existence of a novel type of super cavity solitons.
\end{abstract}

OCIS codes: (230.5750) Resonators; (190.4410) Nonlinear optics, parametric processes; (190.4380) Nonlinear optics, four-wave mixing; (190.4970) Parametric oscillators and amplifiers.

\section{Introduction}

The generation of optical frequency combs in microresonator devices is currently attracting a significant amount of research interest. Microresonators pumped by a continuous wave $(\mathrm{CW})$ laser are known to allow a wealth of dynamical behaviours including both stable and unstable states, periodic temporal patterns and cavity soliton solutions. Microresonator frequency comb sources have a large number of potential applications in e.g. the areas of precision metrology, spectroscopy, optical clocks, wavelength-division multiplexing and arbitrary waveform generation [1].

Studies of frequency comb generation in microresonators have so far been limited mostly to the mean-field regime where the intracavity power is fairly low. The temporal dynamics can then be described either in the time-domain by using the Lugiato-Lefever equation (LLE) [2], or in the frequency domain using the theory of coupled mode equations. However, mean-field models are unable to model the complete range of dynamics that have been observed to occur in nonlinear resonator devices. One example is the period doubling behaviour that has been experimentally observed in dispersive fiber-ring cavities when pumped by a synchronized train of high intensity pulses $[3,4]$.

In this presentation we report on a theoretical and numerical study of frequency comb generation beyond the meanfield approximation and the LLE. Particularly, we consider microresonators where the nonlinear phase-shift is relatively large (of the order of unity). It is found that new dynamical regimes appear at high intracavity power, including multi-stable stationary CW states and a novel type of mode-locked super cavity solitons.

\section{Ikeda map}

To model frequency comb generation without resorting to the mean-field approximation, we use the dispersive Ikeda map [5] as our model framework. The Ikeda map relates the slowly varying field envelope $E^{m+1}(t, z=0)$ at the beginning of roundtrip $m+1$ to the field at the end of the previous roundtrip $E^{m}(t, z=L)$ and the external pump field $E_{i n}$. The evolution of the field within the waveguide of length $L$ is described by a nonlinear Schrödinger equation, viz.

$$
\begin{gathered}
E^{m+1}(t, 0)=\sqrt{\theta} E_{i n}+\sqrt{1-\theta} e^{i \phi_{0}} E^{m}(t, L), \\
\frac{\partial E^{m}(t, z)}{\partial z}=-\frac{\alpha_{i}}{2} E^{m}(t, z)-i \frac{\beta_{2}}{2} \frac{\partial^{2} E^{m}(t, z)}{\partial t^{2}}+i \gamma\left|E^{m}(t, z)\right|^{2} E^{m}(t, z) .
\end{gathered}
$$

Here $t$ is time and $z$ is the longitudinal coordinate along the waveguide, while $\theta$ is the coupling transmission coefficient, $\phi_{0}=2 \pi l-\delta_{0}$ is the linear phase-shift, with $\delta_{0}$ the pump frequency detuning, $\alpha_{i}$ is the intrinsic absorption, $\beta_{2}$ is the second order dispersion coefficient and $\gamma$ is the nonlinear coefficient.

The Lugiato-Lefever equation can be obtained by averaging the above map Eq.(1-2), over one roundtrip time $t_{R}$ and replacing the longitudinal coordinate with a slow evolution time $\tau$, see [2,6], viz. 


$$
t_{R} \frac{\partial E(t, \tau)}{\partial \tau}+i \frac{\beta_{2} L}{2} \frac{\partial^{2} E(t, \tau)}{\partial t^{2}}-i \gamma L|E(t, \tau)|^{2} E(t, \tau)=-\left(\alpha+i \delta_{0}\right) E(t, \tau)+\sqrt{\theta} E_{\text {in }}
$$

The LLE is consequently a less general description for the dynamics of the cavity. We discuss the necessary conditions for the validity of the mean-field approximation [6], and show that while the LLE is an excellent approximation when the characteristic nonlinear length scale is much longer than the path length of the cavity, the approximation starts to break down as the two become comparable. Physical parameters necessary for observing large nonlinear phase-shifts are considered, and we predict that new dynamical regimes could be observable in appropriately engineered highly nonlinear semiconductor microring resonators for sufficiently large input pump powers. We also show examples from the literature of other types of microresonators where large nonlinear phase-shifts have already been experimentally demonstrated.

\section{Multi-valued stationary states and stability analysis}

We perform an analysis of the Ikeda map and demonstrate that is has multi-valued stationary CW solutions, including additional high intracavity power solutions not present in the LLE. The intracavity power is found to satisfy the following relation

$$
\frac{1}{(1-\rho)^{2}+4 \rho \sin ^{2}(\phi / 2)}=\frac{|E|^{2}}{\theta\left|E_{i n}\right|^{2}}, \quad \phi=\delta_{0}-\gamma L_{e f f}|E|^{2} .
$$

Figure 1 shows a comparision of stationary CW solutions of Eq.(4) for the Ikeda map and the mean-field limit of the LLE. Surprisingly, we find that the high power solutions do not necessarily have to correspond to large pump powers, and that higher-order multi-stable states may even by present simultaneously as the low-power bistable response of the LLE.

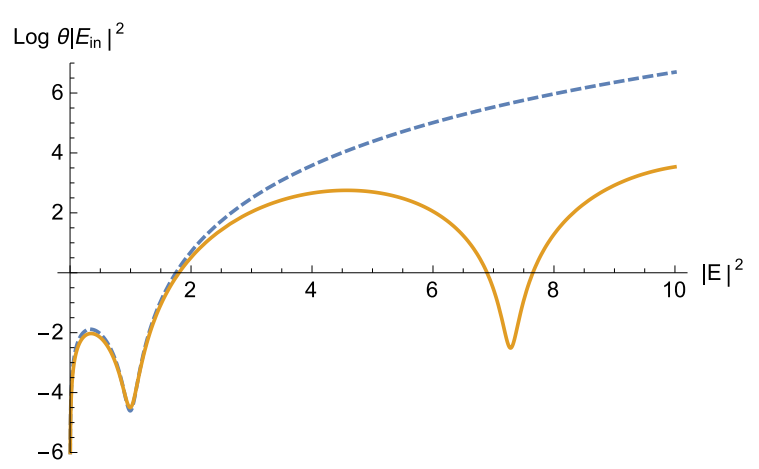

Fig. 1. Comparison of pump dependence on the intracavity power for multi-valued stationary continuous wave solutions of the LLE (dashed blue) and the Ikeda map (solid orange).

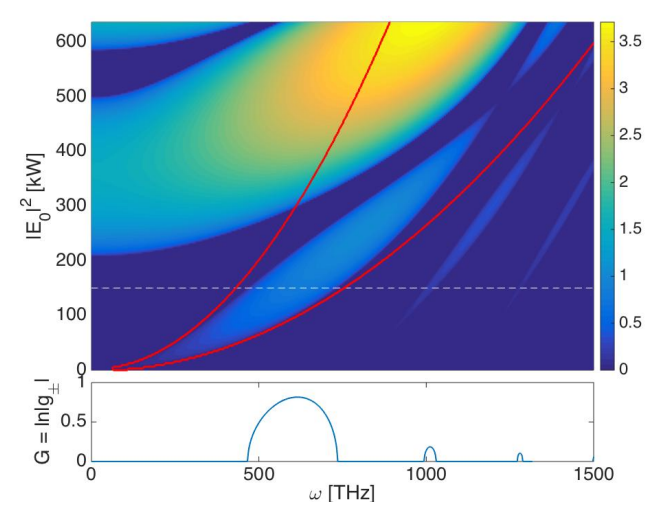

Fig. 2. Parametric instability tongues of the Ikeda map for anomalous dispersion. The red contour shows the predicted range of modulational instability for the LLE. Below a cross section corresponding to the dashed line is shown with alternating CW-MI/P2-MI gain bands.

Additionally,we investigate the modulational stability of the Ikeda map both analytically and numerically by using Floquet theory. We show that new instability gain bands (resonance tongues) appear at high intracavity power due to the presence of cavity boundary conditions. An example for parameters corresponding to a high finesse toroidal silica resonator is shown in Fig. 2. Instabilities having either the fundamental roundtrip period (CW-MI) or period doubling instabilities (P2-MI) are found to appear, see Ref. [7]. The latter instability corresponds to nonlinear waves that repeat themselves not with the fundamental period of one roundtrip but every other roundtrip. The period doubling instability is found to be particularly important for normal dispersion cavities where it is often the first instability to appear as the pump power is increased. Examples are given of how the period doubling instability can give rise to an alternating temporal pattern of two identical but out of phase solutions. 


\section{Super cavity solitons}

Finally, we demonstrate the possibility of creating a novel type of super cavity soliton in microresonator devices. The super cavity solitons are localized dissipative structures that are associated with the higher-order multi-stable states of the Ikeda map, and that cannot be predicted using the LLE. For appropriate parameters we find that super cavity solitons may even coexist simultanously with the LLE soliton for the same pump parameters. An example of two different types of cavity soliton solutions found in the same resonator for identical pump power and detuning is shown in Figs. 3-4. The super cavity solitons are found to exist for relatively low pump power, and are seen to be far more energetic than their LLE counterparts. They have both a larger peak power and are also narrower than the ordinary cavity soliton. As a consequence their spectrum is broader, with intriguing possibilities for the efficient generation of mode-locked octave spanning Kerr frequency combs.
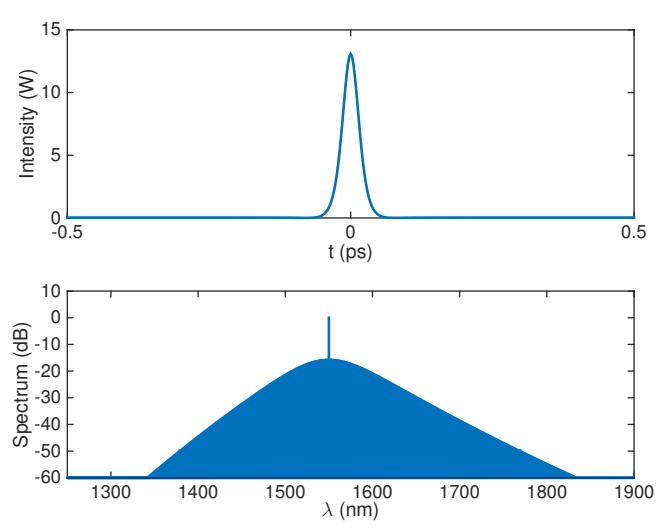

Fig. 3. LLE cavity soliton with background level $|E|^{2} \approx 0.029 \mathrm{~W}$ and support level $|E|^{2} \approx 6.365 \mathrm{~W}$. Numerical simulation of Ikeda map Eqs.(1-2).
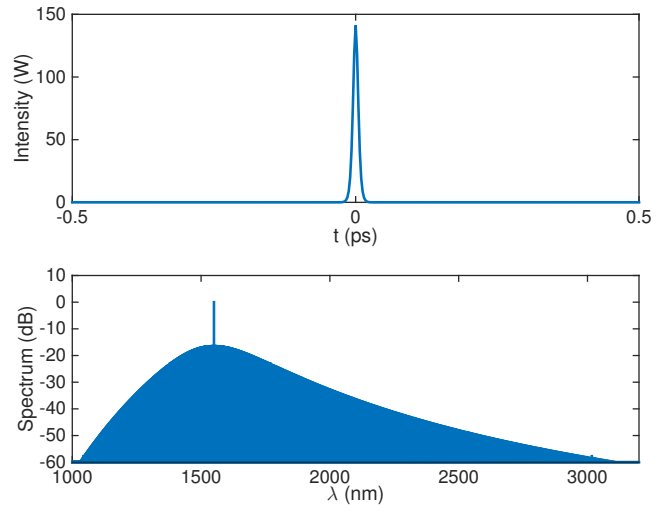

Fig. 4. Stationary super cavity soliton with background level $|E|^{2} \approx 0.029 \mathrm{~W}$ and support level $|E|^{2} \approx 68.764 \mathrm{~W}$, using the exact same parameters as in Fig. 3 except for different initial conditions.

\section{References}

1. T. J. Kippenberg, R. Holzwarth, and S. A. Diddams, Microresonator-Based Optical Frequency Combs, Science 332, 555-559 (2011)

2. S. Coen, H. G. Randle, T. Sylvestre, and M. Erkintalo, Modeling of Octave-Spanning Kerr Frequency Combs using a Generalized Mean-Field Lugiato-Lefever Model, Opt. Lett. 38, 37-39 (2013)

3. R. Valle, Temporal instabilities in the output of an all-fiber ring cavity, Opt. Comm. 81, 419-426 (1991)

4. S. Coen, M. Haelterman, P. Emplit, L. Delage, L. M. Simohamed, and F. Reynaud, Experimental investigation of the dynamics of a stabilized nonlinear fiber ring resonator, J. Opt. Soc. Am. B 15, 2283-2293 (1998)

5. K. Ikeda, Multiple-valued stationary state and its instability of the transmitted light by a ring cavity system, Opt. Comm. 30, 257-261 (1979)

6. M. Haelterman, S. Trillo, and S. Wabnitz, Dissipative modulation instability in a nonlinear dispersive ring cavity, Opt. Comm. 91, 401-407 (1992)

7. S. Coen and M. Haelterman, Modulational instability induced by cavity boundary conditions in a normally dispersive optical fiber, Phys. Rev. Lett. 79, 4139-4142 (1997) 